\title{
ON THE USE OF PARTICLE-IN-CELL METHODS FOR THE STUDY OF MAGNETICALLY-CONFINED FUSION PLASMAS
}

\author{
Richard J. Procassini \\ Massively Parallel Computing Initiative \\ Lawrence Livermore National Laboratory \\ Livermore, CA 94550
}

and

\section{Electronics Research Laboratory \\ University of California \\ Berkeley, CA 94720}

\section{June 1991}

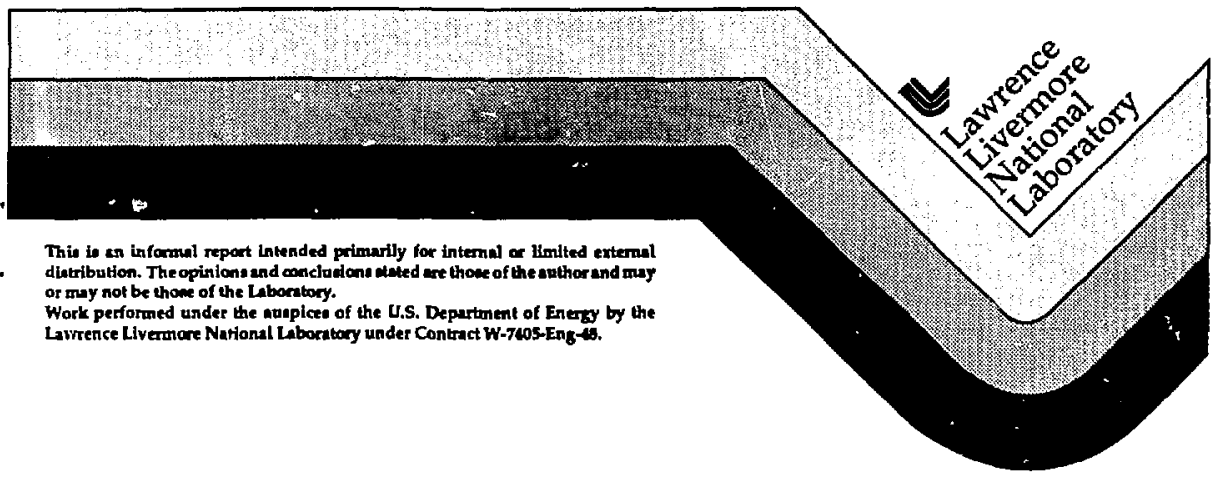




\section{DISCLAIMER}

This document was prepared as an account of work sponsored by an agency of the United States Government. Neither the United States Government nor the Universily of California nor any of their employees, makes any warranty, express or implied, or assumes any legal liability or responsibility for the accuracy, completeness, or usefulness of any information, appasatus, product, or process disclosed, or represents that its use would not infringe privately own rights. Reference herein to any specific commercial products, process, or service by trade name, trademark, manufacturer, or otherwise, does not necessarily constitute or umply its endorsement, recommendation, or favoring by the United States Government or the University of California. The views and opinions of authors cxpressed herein do not necessarity state or reflect those of the United States Goverument or the University of California, and shall not be used for advertising or product endorsement purposes.

This report has been reproduced directly from the best available copy.

Available to DOE and DOE contractors from the Office of Srientific and Techrical Intormation

P.O. Box 62, Oak Ridge, TN 37831

Prices available from (615) 576-8401, FTS 626-8401

Available to the public from the National Technical Information Service

U.S. Department of Commerce 5285 Port Royal Rd. Springfield, VA 22161 


\title{
On The Use Of Particle-In-Cell Methods For The Study of Magnetically-Confined
}

\section{Fusion Plasmas}

UCRL-ID--107220

DE91 015093

\author{
Ricliard J. Procassini \\ Massively Parallel Compuling Initiative \\ Lawnence Livermare National Laloratory \\ Livermore, CA D\$550 \\ and \\ Elcctronics Research Laboratory \\ Iniversity of Culifornia \\ Berkeley, CA $9 / 720$
}

12 June 1991

\begin{abstract}
The applicability of electrostatic particle-in-cel' (JIC) methods for the simulation of magnetica!ly-confined fusion plasinas is investigated. The aspects of the PIC methodology which allow one to accurately model lie representatjve tharge separations found ju hot fusion plass!as with far fewer simulation particles arc discussed. The number of simulation pitrticles recpuired to resolve the collective eflects of iaterest (such as the ambijolitr potential) above the statistical fluctuations is also analyzed.
\end{abstract}




\section{Introduction}

Particle simulations of magnetically-confined fusion plasmas usially employ on the order of $10^{4}-10^{6}$ simulation particles to represent high density plasuss with $n_{e} \simeq n_{i} \geq 10^{14} \mathrm{~cm}^{-3}$ : occupying volumes of many cubic centimeters to cubic meters. Hence, while the cliarge to mass ratio may be the same in the laboratory and computer experiments, each of the simulation particles, or superparticles, has a mass and charge that are several orders of magnitude larger than the physical mass and charge of an electron or ion. The larger charge associated with a superparticle, and the smaller number of superparticles that are used to simulate the warm fusion plasma, lead to an increased level of statistical fluctuations of the electrostatic potential. These fluctuations in density about a mean value vary $\sim 1 / \sqrt{N_{V}}$, where $N_{V}$ is the number of superparticles in the volume $V$ under consideration. The particle-in-cell (PIC) techniçues which allow one to model a warm fusion plasma accurately with a limited number of simulation particles, and the criteria that must be met to ensute that the level of the statistical fluctuations is smaller than the clesired collective effects are the subjects of this paper. Some of the important advances in the area of particle simulation of thermal plasmas are reviewed in Section 1. In Section 2 we discuss the methods by which a limited number of simulation particles gives rise to the proper charge separation associated with thermal ievel potential variations. The requirements on the number of simulation particles per wavelength for resolution of the potential structure above the statistical fluctations are presented in Section 3.

\section{Particle Simulation Models Of Fusion Plasmas}

Bifferent types of particle simulation methods are applicable to the study of fusion plasmas. The Particle-Particle (PP) method [1], which is based upon the action at a distance formulation of the force law, uses the interparticle spacing to calculate the Cunlonb force on one point-sized particle due to all others. While this method is simple to program, and is capable of simulating both collisonless and highly collisional plasmas, it is computationally expensive, requiring $\mathcal{O}\left(N_{p}\right)$ operations per time step, where $N_{p}$ is the number of particle in the system. In addition, sitntulation of bounded plasma systems with the ple method is not st raightforward. 
One of the earliest warm plasmas models to use the PP simulation method wis the 196i2 one-dimensional model of Dawson [2], which treats the electrons as zero-widt charge shects embedded in a fixed neutralizing background. Dawson's model accuratcly reproduced the thermalizing propertics and stochinstic behavior of periodic, thermal plasmias with densities as low as 5 superparticles per Debye length. Time-averaging was employed to reduce the level of statistical fluctuations that are associated with such a small nuinber of "point" particles.

In contrast to the PP method, Particle-Mesh (PM) methods advance the trajuctory of the particles using field quantities that are defined on a collection of discrete grid points which span the system. The charge density and current contribution of each particle is weighted to the neighboring grid points according to the shape factor $S_{p s}\left(x-X_{j}\right)$, where $x$ is the particle location and $X_{j}$ is the location of the $j-$ th grid point. The charge flensity and current density at each grid point are used to calculate the electric and magnetic field at the grid points. The lorentz force associated witl these fields is interpolated to the particlo location via the shape factor $S_{g p}\left(x-X_{j}\right)$. In general, $S_{p g}$ and $S_{\varphi p}$ may use diJferent order interpolating schemes [3]. PM methods that employed various interpolatiug scitemes were developed in the mid to late 1960 's, most notably the zero-order Nearest Grid Point (NGP) [4], and the first-order Cioud-In-Cell (CIC) [5] and Particle-In-Cell (PIC) [6] models.

The use of a grid and shape factors (usually in the form of iuterpolating splines) results in a particle which has finite extent and is tenuous in nature. These properties of the particles lead to a plasma that more closely resembles a contjnuum flujd, or fuid elements, than the collection of point particles used in the PP method, while retaining some the kinetic nature of point particles, For a given number of particles, PM methods have a lower integrated levels of statistical fluctuations than dous the PP method $[7,8]$. Therefore, PM methods (such as CIC/PIC) allow une to obsain reasonable accuracy with wrikes of magnitude fewer particles per Debye length than occur in the physical system that is being modeled without resorting to special methods that reduce the statistical noist. On thre other hend, PM methods are only capa!sle of resolving forces which are smootily varying over distances that are longer than a grid spacing $\Delta x$. Since some parl of coulomb forces acl over a distance that is much sliorter thin $\Delta x$, it is not possible to simulate the shortrange Coulomb collisional forces via PM methods, witlout explicitly modeling the physics 
of these short-range collisional processes. Fortunately, for many warm plisulias of interest, large-impact-parameter $\left(b \simeq \lambda_{D_{e}}>\Delta x\right.$ ), small-angle Coulomb collisions dominate the short-range, large-scattering-angle collisions by a factor of $\ln \left(N_{D}\right)$, where $N_{D}$ is the number of particles in a cube of length equal to the Debye lengtt: $\lambda_{D}$.

\section{Charge Separation And Electrostatic Potentials In A Particle-In-Cell Code}

The fractional charge separation $\left(n_{i}-n_{e}\right) / n_{e}$ which is required to create a potential variation $e \Delta \phi$ (which is some fraction $f$ of the electron thernal energy $k_{B} T_{c}$ ) over a scale length $\lambda$ may be readily estimated from Poisson's cquation

$$
\nabla^{2} \phi=-4 \pi e\left(n_{i}-n_{e}\right)
$$

where $\phi$ is the electrostais potential, $n_{i}$ and $n_{e}$ are the ton and electron densitics and $e=\left|\varphi_{e}\right|=q_{i}$ is the charge of the ion (i. e. $Z=1$ ). For a one-dimensional potential variation along $\hat{x},(1)$ may be rewritien as

$$
\frac{d^{2} \phi}{d x^{2}}=-4 \pi e\left(n_{i}-n_{e}\right)
$$

whiclı may be approximated by

$$
\frac{\Delta \phi}{\lambda^{2}} \simeq-4 \pi c\left(n_{i}-n_{c}\right)
$$

Multiplying both sjdes of (2) by $\lambda^{2} \approx / k_{B} T_{e}$ gives

$$
\begin{aligned}
\frac{e \Delta \phi}{k_{B} T_{c}} & \simeq \lambda^{2}\left(\frac{4 \pi e^{2} n_{c}}{k_{B} T_{c}}\right)\left(\frac{n_{e}-n_{i}}{n_{e}}\right) \\
& \simeq\left(\frac{\lambda}{\lambda_{D_{e}}}\right)^{2}\left(\frac{n_{e}-n_{i}}{n_{e}}\right)
\end{aligned}
$$

where $\lambda_{D e}$ is the electron Debye length. Setting $e \Delta \phi / k_{B} T_{e}=j$ and rearrangiug gives

$$
\left(\frac{n_{c}-n_{i}}{n_{r}}\right) \simeq \int\left(\frac{\lambda_{D_{e}}}{\lambda}\right)^{2} .
$$

For typical fusion plasmit pammeters of $n_{c} \simeq n_{1} \simeq 10^{14} \mathrm{~cm}^{-3}$ and $k_{B} T$, $\simeq 20 k e V$. the electron Debye length is $\lambda_{0 e} \simeq 10^{-2} \mathrm{~cm}$. For these potential varjations with a scaic 
length $\lambda \simeq 10 \mathrm{~cm}$ (a reasonable value for present magnetically-confined fusion plasmas), the resultant fractional charge separation is approximately

$$
\left(\frac{n_{c}-n_{i}}{n_{e}}\right) \simeq \int \times 10^{-6} \text {. }
$$

The fraction $f$ of the electron thermal energy that is converted to an electrostatic potential is $\approx 1 / N_{D}$, which is usually much less than one, hence $f \ll 1$. Even for $f \simeq 1$, the ex. pected charge imbalance is very small. Therefore, long-wavelength potential variations are associated with very small fractional cliarge separations and the plasina is termed puosinintral. Since many interesting phenomena iı fusion plasnia physies are duasilueutral with long characteristic wavelengt]s, equatiou (5) implies that a simulation of such a plastlia nust resolve fractional density imbalances over the wavelength of interest to better than 1 part in $10^{6}$.

On first reading, the fractional charge separation given by (5) may appcar to imply using more than $10^{6}$ point particles per wavelength. On this basis, one might argue that the PP method is not practical for the simulation of a fusion plasma which spaus several wavelengths. On the other hand, the finite extent of the particles in PM methods aliows one to resolve cliarge separations, whicl are detersnined by the location of the centers of the particles, to witlin the accuracy of the computer. Figure A.1 shows a first-order-weighted $\mathrm{CIC} / \mathrm{PIC}$ finite-size particle in a gridded sysiem at two successive time steps. At time stop $n$ (solid particle shape), the particle $i$ is closer to grid point $j$ than it is to $j+1$, hence the particle's contribution to the charge density at grid point $j\left(\rho_{i, j}\right)$ is larger thian that to grid point $j+1\left(\rho_{i, j+1}\right)$. The particle is advanced to the right in the time step $\Delta t$ by the incremental displacement $\delta$. Therefore, at time step $n+1$, the particle ccitribution to the charge density at grid point $j$ is reduced relative to that at time step $\pi$, and vice versa for the charge density at grid point $j+1$. The resolution of the displacenent $\delta$ is linited only by the accuracy of the computer. Therefore, very small charge inbalances are possible with PM methods, even for a small number of particles per grid cell.

It should be pointed out that the number of superparticles that are required for the simulation of warm plasmas, such as a fusion-grade plasma, is much larger thaul that required to simulate a cold plasma or a beam. For the latter cases, one only needs a sufficient number of particles to reasonably fill in the configuration "axis" of $t]_{3}(x, v)$ phose space. However, simulation of a thermal plasma reguires enough particles to fill in tle pluase space region 


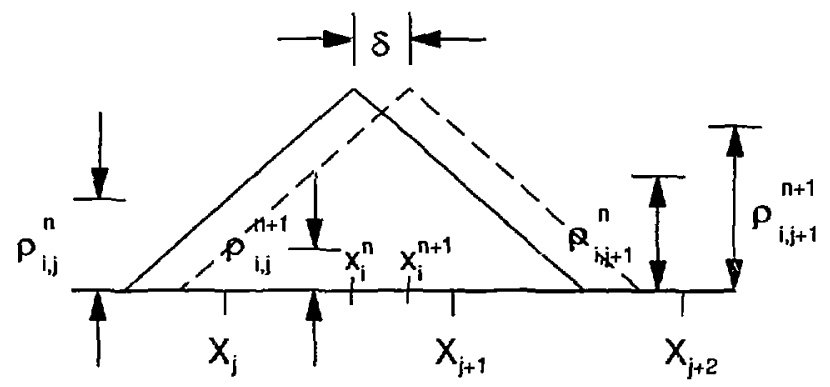

Figure 1: Location of a finite-size, first-order weighted CIC/PIC particle in a gridded system at two successive time steps. The particle is advanced at a velocity $v_{i}$ to the right during the time step $\Delta t$ for an incrimental displacement $\delta$.

from $v_{\min }$ to $v_{\max }$, over the entire length of the system, such that the "grainy" simulation phase space closely resembles the nearly continuous physical phase space.

\section{Statistical Fluctuations And Electrostatic Potentials In A Particle-In-Cell Code}

The goal of particle simulations is to obtain a reasonably accurate description of the plyysical system that is being modeled, with a finite number of superparticles. Since the number of superparticles used in any simulation of a fusion plasma is many orders of magnitude smaller than the aciual number of particles in the physical plasma, the statistical fuctuation level of the simulation plasma is expected to be greater than that for the physical plasma. One Inust ensure that these fluctuations are not larger than the electrostatic potential which is the result of collective effects in the plasma.

Jow many superparticles per Debye length are required to resolve suall changes in the 
potential structure above the statistical fluctuations? The answer may be obtaincd from the general dispersion relation for an electrostatic wave in an unmagnetized, collisionless plasma. The dispersion relation can be derived from the fluid equations for eacis species and Poisson's equation (1). Neglecting sources of particles and momenturn, the fluid expations become

$$
\begin{aligned}
\frac{\partial n_{s}}{\partial t}+\nabla \cdot\left(n_{s} u_{s}\right) & =0 \\
m_{s} n_{s}\left[\frac{\partial \mathbf{u}_{s}}{\partial t}+\left(\mathbf{u}_{s} \cdot \nabla\right) \mathbf{u}_{s}\right] & =-\nabla p_{s}+q_{s} n_{s} \mathbf{E} \\
p_{s}=C p_{s}^{?} & , \nabla p_{s}=\gamma k_{s} T_{s} \nabla n_{s} .
\end{aligned}
$$

Equations (6), (7) and (8) are the particle continuity equation, momentum balance equation and equation-of-state for species $s$ respectively, with $C$ a constant coeflicient. Assunilig small-amplitude, plare-wave $\left(\mathcal{F}(r, t)=\mathcal{F}_{0} \exp (i \mathbf{k} \cdot \mathbf{r}-i \omega t)\right)$ pert urbations, linearizing about an equilibrium $\left(u_{0},=0, \partial n_{0 s} / \partial t=0\right.$ and $\left.\nabla n_{0_{3}}=0\right)$ and substituting ( 8$)$ into ( 7$)$ produces

$$
\begin{aligned}
-i \omega n_{1 s} & +i k \cdot\left(n_{0 s} \mathrm{u}_{1 s}\right)=0 \\
-i \omega m_{s} n_{0 s} \mathrm{u}_{1 s} & =-\gamma k_{\theta} T_{s} i \mathrm{k} n_{1 s}-i k \eta_{s} n_{0 s} \phi_{1} \\
k^{2} \phi_{1} & =4 \pi e\left(n_{1 i}-n_{1 e}\right)
\end{aligned}
$$

where (9) is the continuity equation and $(10)$ is the monentum efjualion for species $s$, and (11) is Poisson's equation. It is assumed that $\left|q_{e}\right|=q_{i}=c$. Solving (9) and (10) for the perturbed density gives

$$
n_{1 s}=\frac{n_{0 s} k^{2} e \phi_{1}}{n_{g} \omega^{2}-\gamma k_{B} T_{s} k^{2}}
$$

which are inserted in to (11) to obtain

$$
k^{2} \phi_{L}=4 \pi n_{0} e^{2} k^{2} \phi_{L}\left[\frac{1}{m_{i} \omega^{2}-\gamma} \frac{1}{k_{B} T_{i} k^{2}}-\frac{1}{m_{e} \omega^{2}-\gamma k_{B} T_{t} k^{2}}\right]
$$

which, after cancelling terms and rearranging, becomes

$$
1=\left(\frac{\omega_{p e}}{\omega}\right)^{2}\left[\frac{m_{e}}{m_{i}-\frac{\gamma k_{B} T_{i} k^{2}}{\omega^{2}}}-\frac{m_{e}}{m_{e}-\frac{\gamma k_{B} T_{e} k^{2}}{\omega^{2}}}\right] .
$$

The definition of the electron plasina frequency $\omega_{p e}^{2} \equiv 4 \pi e^{2} n_{0} / m_{\mathrm{c}}$ las been used ill derivilug (13), whers $n_{0 e}=n_{0 i}=n_{0}$ is the unperturbed density. Using the defitition of the Debye 
lengih $\lambda_{D} \equiv v_{t h} / \omega_{F}=\sqrt{k_{B} T / m} / \omega_{p}$, assuming that the plasma is isothermal $\left(k_{B} T_{e}=\right.$ $k_{B} T_{i}=k_{B} T$ ) and rearrangirg gives

$$
1=\left(\frac{\omega_{p e}}{\omega}\right)^{2}\left[\frac{1}{\frac{m_{i}}{m_{e}}-\frac{\gamma \lambda_{D}^{2} k^{2} \omega_{p c}^{2}}{\omega^{2}}}-\frac{1}{1-\frac{\gamma \lambda_{D}^{2} k^{2} \omega_{p e}^{2}}{\omega^{2}}}\right] .
$$

Since quasineutral effects are of interest, examine (14) in the low-frequency ( $\omega \ll \omega_{p e}$ ). long-wavelength $\left(k \lambda_{D}<1\right)$ limit, where

$$
\left(\frac{k \lambda_{D} \omega_{p e}}{\omega}\right)^{2} \gg \frac{m_{i}}{m_{c}}
$$

and

$$
\left(\frac{k \lambda_{D} \omega_{p c}}{\omega}\right)^{2} \gg 1
$$

In the quasineutral limit, the dispersion relation reduces to

$$
\begin{aligned}
1 & \simeq \frac{1}{\gamma}\left(\frac{\omega_{p e}}{\omega}\right)^{2}\left[-\left(\frac{\omega}{k \lambda_{D} \omega_{p e}}\right)^{2}+\left(\frac{\omega}{k \lambda_{D} \omega_{p e}}\right)^{2}\right] \\
& \simeq \frac{1}{\gamma}\left[-\left(\frac{1}{k \lambda_{D}}\right)^{2}+\left(\frac{1}{k \lambda_{D}}\right)^{2}\right] .
\end{aligned}
$$

Equation (i5) indicates that the Laplacian of the perturbed potential $\nabla^{2} \phi_{1}$ is balanced by the difference of the nearly equal species densitjes $n_{1 e}$ and $n_{1 i}$, and that eacil of the density terms is of order $\left(1 / k \lambda_{D}\right)^{2} \gg 1$ times greater than the laplacian term.

The level of the statistical fluctuations may be quantified by the error associated with the process of counting particies to produce the densities $n_{1}$ and $n_{1 i}$. For a one-dimensional perturbation, the standard deviation about the mean perturbed density $\left(n_{1}=N_{A} / \lambda\right)$ of ejther species is defined as

$$
\sigma_{n_{1}} \equiv \frac{n_{1}}{\sqrt{N_{\lambda}}}=\left(\frac{N_{\lambda}}{\lambda}\right) \frac{1}{\sqrt{N_{\lambda}}}=\frac{1}{\lambda} \sqrt{N_{\lambda}}
$$

where $\lambda$ is the wavelength of the rlensity perturbations and $N_{\lambda}$ is the number of particles per wavelength. Since the perturbed densities are proportional to $\left(k \lambda_{D}\right)^{-2}$ from $(15)$, the statistical fluetuations in the perturbed density and potential scale as

$$
\sigma_{n_{1}} \sim \frac{1}{\sqrt{N_{1}}}\left(\frac{1}{R \lambda_{D}}\right)^{2}
$$


It is desirable to have the signal to noise (statistical Ructuations) ratio be as large as possible, i. e. $\sigma_{n_{1}} \ll 1$, such that

$$
N_{\lambda} \gg\left(-\frac{1}{k \lambda_{D}}\right)^{4}
$$

For long-wavelength perturbations $\left(1 / k \lambda_{D}\right)>1$, equation (18) appears so be a rather severe requirement on the number of parlicles per wavelength that are required to obtait accurate results with reasonable statistical fluctuations. Note that this derivation assumes that the particles are effectively point sized. The finite extent of the particles used in CIC/PIC simulations leads to a lower integrated level of fluctuations than that encountered in simulations which use the PP method, since wavelengths shorter thin the size of a particle are attenuated by the finite extent of the particle. For this reason, equation (18) may be taken as an upper limit on the number of particles required per wavelength in a $(: \mathrm{JC} / \mathrm{PlC}$ simulation.

Equation (18) also delermines the required number of particles per Debye fength, since $k=2 \pi / \lambda$. Fusions plasmas have approxinately $10^{8}$ particles per Debye cube it three dimensions $\left(N_{D} \equiv n_{3 D} \lambda_{D}^{3} \simeq 10^{8} \gg 1\right.$ ). By reducing the system from three dinensions in configuration space down to onc, the number of particles per Debye lengtli is $N_{D} \simeq$ $\left(n_{3 D} \lambda_{D}^{3}\right)^{1 / 3} \simeq 500$, and the relation $N_{D} \gg 1$ still Holds.

Simulations of long-wavelength phenomena using the PP method requires both $N_{\lambda} \gg 1$ and $N_{D} \gg 1$. For a PM method simulation using CIC/PIC techniqnes, tlte grid (and resulting finite-sized particles) eliminate perturbations with wavelengths hat arc shorter than $2 \Delta x$. In this case, the parameter $\Delta x / \lambda_{D}$ becomes important. If $\Delta x / \lambda_{D} \ll 1$, the number of particles required to simulate phenomena with waveiengths on the order of a fow grid spacings is much less than $N_{D}$, but the $N_{D} \gg 1$ constraint is still valid. If $1 x / \lambda_{U} \sim 1$. then only $\sim N_{D}$ particles are needed to simulate the shortest wavelength plinsonicha. $A s$ $\Delta x / \lambda_{D}$ is increased above 1 , the Debye length is no longer resolved, and $N_{.,}$replaces $N_{D}$ as the important linear density. When $\Delta x / \lambda_{D} \gg 1$, wavelengths on the order of the Delyye length are unresolvable, allowing une to reduce the number of particles per Debye length to $N_{D} \sim 1$. In the large grid cell linit, the required number of particles per wavelength gi zen by $(18)$ is replaced by

$$
N_{\lambda}>\left(\frac{1}{k \Delta x}\right)^{4}
$$


In each of these limits, the constraint $N_{\lambda} \gg$ I still holds.

Since implicit formulations of the CIC/PIC method allow one to run at large values of $\omega_{p e} \Delta t$ and $\Delta x / \lambda_{D e}$ without incurring numerical instabilities, the total number of particles that are required to simulate long-wavelength or quasineutral phenomena (at the ër,ense of damping out the high-frequency response) is significantly reduced relative to the number required by explicit schemes, which lave numerical instability limits on the size of the time step and grid spacing. For tris reason, implicit schemes are more attractive than explicit schenes for the simulaiion of large-scale, quasineutral fusion plasmas.

\section{Acknowledgments}

The author gratefully acknowledges discussions with Professor C. K. Birdsall (U Viversity of California ấ Berkeley) and Dr. B. I. Colıen (Lawrence Livermore Nationa) Laboratory). This paper attempts to answer the question posed by Professor T. K. Fowler (University of California at Berkeley) during the presentation of the author's dissertation researcl proposal. This research was funded by a grant from the Plasma Physics Research Institute of the Lawrence Ljvermore National Laboratory. Additional funding was provided to the Plasna Theory and Simulation group at the University of California at Herkejey by the U. S. Department of Energy under Contract Number DÉ-FG03-86ER53220. 


\section{References}

[1] R. W. Hackney and J. W. Eastwood, Computer Simulation Using Paricles, McGrawHill, New York, Pp. 18-22 (1981).

[2] J. M. Dawson, Phys, Fluids 5, 445 (1962).

[3] C. K. Birdsall and A. B. Langdon, Plasma Physics via Compuler Simulation, McGiraw. Hill, New York, pp. 162-164, 168-1:0 (1985).

[4] R. W. Hockney, Phys. Flsids 9, 1826 (1966).

[5] C. K. Birdsall and D. Fuss, J. Comput. Phys. 3, 494 (1969).

[6] R. M. Marse and C. W. Nielson, Hhys. Fluids 12, 2418 (1969).

[7] A. B. Langdon and C. K. Birdsall, Phys. Fluids 13, 2115 (1970).

[8] T. Tajima, Computational Plasma Physics With Applications to Fusion and Astrophysics, Addison-Wesley, New York, pp. 46-49 (1089). 